\title{
Stellar wind bubbles around WR and [WR] stars
}

\author{
G. Mellema ${ }^{1}$ and P. Lundqvist ${ }^{2}$ \\ 1 Sterrewacht Leiden, PO Box 9513, 2300 RA, Leiden, The Netherlands \\ e-mail: mellema@strw.leidenuniv.nl \\ ${ }^{2}$ Stockholm Observatory, AlbaNova (SCFAB), Department of Astronomy, 10691 Stockholm, Sweden \\ e-mail: peter@astro.su.se
}

Received 30 April 2002 / Accepted 7 August 2002

\begin{abstract}
We study the dynamics of stellar wind bubbles around hydrogen-deficient stars using numerical simulations with time- and ion dependent cooling. We consider two types of hydrogen-deficient stars, massive WR stars, producing Ring Nebulae, and low mass [WR] stars, producing Planetary Nebulae. We show that for the Planetary Nebulae, the different cooling properties of the hydrogen-deficient wind lead to a later transition from momentum- to energy-driven flow, which could explain the observed turbulence of these nebulae. We find that Ring Nebulae should all be energy-driven, and show how comparing the bubble's momentum and kinetic energy to the input wind momentum and kinetic energy, can give misleading information about the dynamics of the bubble.
\end{abstract}

Key words. planetary nebulae: general - ISM: bubbles - stars: Wolf-Rayet - stars: AGB and post-AGB - hydrodynamics

\section{Introduction}

Both high and low mass stars can under certain circumstances reduce the hydrogen content of their atmospheres. In most cases this leads to the so-called Wolf-Rayet (WR) phenomenon, i.e. a dense fast wind starting below the photosphere, which produces a Wolf-Rayet spectrum, dominated by bright emission lines (Abbott \& Conti 1987).

Traditionally most attention was given to the massive WR stars, but the last ten years their lower mass cousins, the [WR] stars have been studied in more detail (see e.g. Koesterke 2001, for a review).

The winds from [WR] and WR stars produce stellar wind bubbles (SWBs) in their environment. In the case of WR stars they are called Ring Nebulae (RNe), in the case of [WR] stars, Planetary Nebulae (PNe). PNe also form around H-rich central stars, so [WR] stars constitute a subgroup among central stars of PNe. Approximately $7 \%$ of central stars is estimated to be [WR], the rest being H-rich (Górny 2001) (with the exception of so-called weak emission line stars or wels which appear to be H-poor without showing the WR phenomenon). All central stars are considered to be in the same evolutionary phase, namely the post-AGB phase, where the [WR] have changed their atmospheric abundances through a timely thermal pulse (Herwig 2001). The existence of two different groups of central stars suggests that a comparison between the two could be interesting.

Send offprint requests to: G. Mellema, e-mail: mellema@strw.leidenuniv.nl
In contrast, WR stars are thought to be a phase in the evolution of most stars with Zero Age Main Sequence masses higher than $\sim 25 M_{\odot}$, and there is no class of $\mathrm{H}$-rich stars in an equivalent evolutionary stage. Massive stars lose mass already on the Main Sequence, followed by a slower wind when the star moves over to the red part of the Hertzsprung-Russell diagram, and finally leading to the WR wind. This leads to a whole series of interactions between the wind phases (see e.g. GarciaSegura et al. 1996b). The more complicated environments and probably also the clumpiness of the actual winds make that the $\mathrm{RNe}$ are mostly irregular and filamentary, lacking the overall symmetries found in PNe.

In this paper we investigate whether the fact that the winds from WR and [WR] stars are H-poor changes the dynamics of their SWBs. In the case of the [WR] stars this is relevant because we can compare the PNe between the H-rich and $\mathrm{H}$ poor central stars. In the case of the WR stars this is relevant because it has been suggested that even at high wind velocities their SWBs can be strongly cooling.

The layout of the paper is as follows. The effects of cooling on SWBs are outlined in Sect. 2. We investigate the effects of WR winds using numerical hydrodynamic models with detailed cooling, described in Sect. 3. Sections 4 and 5 contain the results of the simulations for $\mathrm{PNe}$ and $\mathrm{RNe}$, respectively. We discuss these results further in Sect. 6 and sum up the conclusions in Sect. 7.

\section{Stellar wind bubbles}

Stellar wind bubbles (SWBs) expanding into a dense environment (as is the case in both $\mathrm{RNe}$ and $\mathrm{PNe}$ ), come in two basics 
types: radiative (also called momentum-driven or momentumconserving) and non-radiative (also called adiabatic or energydriven/energy-conserving). Whether the SWB is radiative or non-radiative depends on the cooling at the wind shock (or inner shock). This is a well-known phenomenon, see for example Dyson \& Williams (1997) or Koo \& McKee (1992a, 1992b).

Since cooling strongly depends on the metallicity, it is expected that the radiative cooling rate of the metal-rich WR winds will be orders of magnitude above normal ("solar abundance") cooling rates. This could then lead to SWBs remaining radiative when they would normally be non-radiative.

Whether a SWB is radiative or non-radiative depends on three time scales (Koo \& McKee 1992b): the crossing time for the free wind $t_{\mathrm{cross}}=R_{\mathrm{sw}} / v_{\mathrm{w}}$, the age of the bubble $t$, and the cooling time $t_{\text {cool }}$. The cooling time can be found from

$$
t_{\text {cool }}=\frac{C_{1} v_{\mathrm{sw}}^{3}}{\rho_{\text {pre }}}
$$

where $C_{1}$ is a constant which describes the cooling, which is normally taken to be $6.0 \times 10^{-35} \mathrm{~g} \mathrm{~cm}^{-6} \mathrm{~s}^{4}$. If $t_{\text {cool }} \ll t_{\text {cross }}$ the bubble is radiative, if $t \ll t_{\text {cool }}$ the bubble is non-radiative, and if $t_{\text {cross }} \ll t_{\text {cool }} \ll t$ cooling does affect the shocked wind, but the bubble is still mostly filled with hot shocked gas, and the bubble is partially radiative.

The application of these expressions lead to evolutionary sequences for stellar wind bubbles, as explored by Koo \& McKee (1992b) for the most general case, by Garcia-Segura \& Mac Low (1995) for RNe, and by Kahn \& Breitschwerdt (1990) for PNe. Here we limit ourselves to the interaction of constant or evolving winds interacting with a previous slow wind phase, the generic model for both PNe and RNe. When a volume of hot shocked wind material is present, we will call the bubble energy-driven, if not, we will call it momentumdriven. This means that a partially radiative bubble will also be considered to be energy-driven.

\section{Numerical method}

We study the formation of SWBs using a numerical hydrodynamics code coupled to a detailed atomic physics module, which calculates the ionization/recombination and cooling for the ions included. The hydrodynamic Euler equations are solved in one spatial dimension using the Roe-solver (Roe 1981; Mellema et al. 1991). The spatial coordinate is radius. The Roe solver solves the equations conservatively, and with second order accuracy in both space and time. The ionic/atomic concentrations are passively advected using the approach from CLAWPACK (LeVeque 2002). This advection scheme was tested using the test problems described in Plewa \& Müller (1999) and found to perform as well as the approach described by those authors.

\subsection{Atomic physics module}

Radiative cooling is due to excitation and ionization of molecules, atoms and ions, and therefore depends on the composition of the gas. Most numerical simulations which include cooling, use a so-called cooling curve. This is a curve which gives cooling as a function of temperature. The first cooling curves were constructed in the sixties, and the one which became standard were done around 1970 by Cox and collaborators (Cox \& Tucker 1969; Cox \& Daltabuit 1971; Raymond et al. 1976). These curves are calculated assuming collisional ionization equilibrium to fix the ion concentrations. Although the original publications contained cooling curves for individual elements (but not ions), the most common use of them has been in the form of one total cooling curve for standard abundances, such as the one from Dalgarno \& McCray (1972). For cosmological applications, $\mathrm{H}$ and He only cooling curves have been constructed; Sutherland \& Dopita (1993) present cooling curves for a range of metallicities. All of the above, as well as our method below, assume an optically thin plasma, in which all of the radiation escapes.

Primarily motivated by astrophysical situations in which photo-ionization (and not collisional ionization) determines the ion concentrations, we have developed numerical approaches in which cooling is calculated for each ion separately and timedependently, using the local temperature and density. The first version of this was described by Frank \& Mellema (1994) and Balick et al. (1993), and a newer version by Raga et al. (1997) and Mellema et al. (1998).

Here we use an improved version of the same approach as in Raga et al. (1997), and Mellema et al. (1998). The improvements are that we now allow for position-dependent elemental abundances, which is crucial for the problem studied here. We also now include all ions of the following elements: $\mathrm{H}, \mathrm{He}, \mathrm{C}$, $\mathrm{N}, \mathrm{O}$ and Ne. Collisional excitation for the highest ionization stages were taken from Gaetz \& Salpeter (1983). We have also included free-free and free-bound cooling to allow an extension to $10^{9} \mathrm{~K}$ using the method of Gronenschild \& Mewe (1978). Free-free Gaunt factors are from Karzas \& Latter (1961), and relativistic free-free corrections from Gould (1980).

Furthermore, we dropped the special treatment of $\mathrm{H}$ and $\mathrm{He}$, which are now treated in the same way as the other elements. Since abundances can vary, we let all elements contribute to the electron density. Cooling due to collisional ionization is currently not included. This will underestimate the cooling at lower shock velocities. The module as used for the results in this paper does not contain photo-ionization, since we are primarily interested in the effects of the H-deficient winds.

Our module does not include any heavier elements than $\mathrm{Ne}$. At the high temperatures where the elements included become completely ionized, heavier elements can start to contribute to the cooling. We therefore underestimate the radiative cooling in the temperature regime $\log _{10} T=6$ to 6.8 , beyond which the free-free starts to dominate (see e.g. Gaetz \& Salpeter 1983). Whether or not this is a serious underestimate of the cooling depends on the relative abundances of the cooling elements. In our simulations it will mostly affect the solar abundances case. We will comment further on this below.

In Fig. 1 we show three equilibrium cooling curves calculated with the DORIC atomic physics module. One for solar abundances, one for primordial abundances, and one for [WC] abundances. These curves illustrate the dramatic effect of abundances on the cooling. Note that the high cooling rate below 


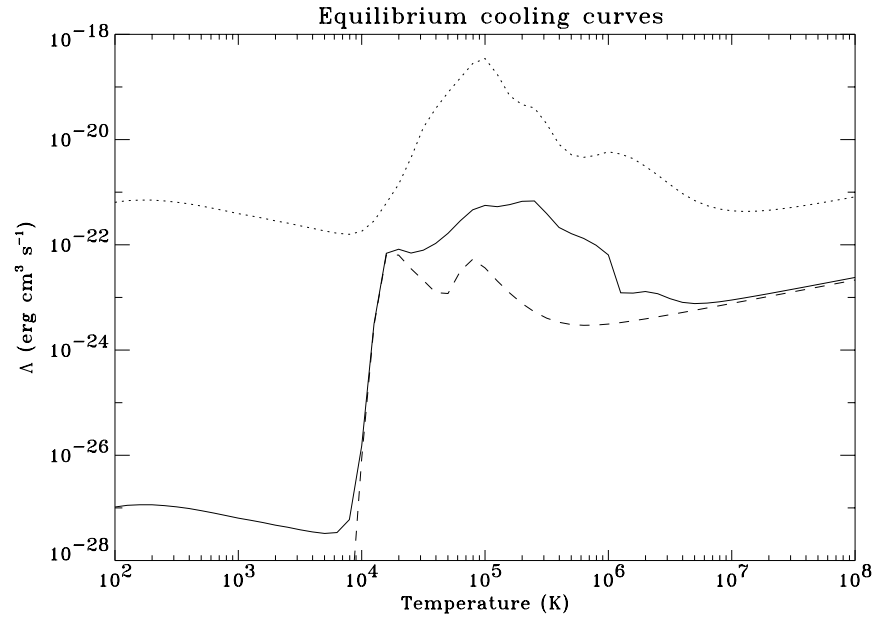

Fig. 1. Collisional equilibrium cooling curves calculated with the DORIC module. Solid line: solar abundances, dashed line: metal free, dotted line: [WC] abundances. The curves are not used in any of the calculations, but are shown to illustrate the effect of metallicity and the capability of DORIC.

$10^{4} \mathrm{~K}$ is entirely due to $\mathrm{CII}$, since this ion is assumed never to recombine (due to a $\mathrm{CI}$ ionizing background).

In the calculations below, we do not use these curves, but instead calculate the concentrations of ions (and therefore the radiative cooling) time-dependently. Especially in low density areas the ionic concentrations can deviate from the equilibrium values. We comment on this in Sect. 5.

\section{Models for PNe}

The properties of [WR] stars have been well studied, and the results from two independent groups agree well. For an overview see the reviews of Koesterke (2001) and Hamann (2002). All [WR] stars are carbon-rich, so from now on we refer to them as [WC] stars. What appears to be an evolutionary sequence of [WC] late, to [WC] early, to the PG 1159 stars, is well sampled, and the abundances are more or less the same all along this sequence; a previous discrepancy has now been resolved, (De Marco, private communication).

The stellar atmosphere analysis yields mass loss rates and velocities. The results show that the wind velocities are similar to the ones from normal central stars, but the mass loss rates are $\sim 10$ times higher. Therefore, we will use a higher mass loss rate in our [WC] model.

To study the effects of H-deficient [WC]-type winds on the formation of $\mathrm{PNe}$, we use the approach from Kahn \& Breitschwerdt (1990) and Dwarkadas \& Balick (1998). The evolution of the stellar wind in the post-AGB phase is not well known, not for normal, nor for [WC] central stars. Hence a simple power law behaviour is postulated. Following Dwarkadas \& Balick (1998) the fast wind evolves according to

$v_{\mathrm{fw}}=v_{\mathrm{fw}}(0)\left(1+\frac{t}{\tau}\right)^{\alpha}$.

The initial velocity $v_{\mathrm{fw}}(0)$ is taken to be $25 \mathrm{~km} \mathrm{~s}^{-1}, \alpha=1.5$, and $\tau$ is chosen such that at $t=3000$ years, $v_{\mathrm{fw}}=2000 \mathrm{~km} \mathrm{~s}^{-1}$.
The mass loss changes so that the product $\dot{M}_{\mathrm{fw}} v_{\mathrm{fw}}$ remains constant. This is expected for a radiation-driven wind (of constant efficiency).

The environment is taken be a slow AGB wind characterized by a constant mass loss rate of $2 \times 10^{-5} M_{\odot} \mathrm{yr}^{-1}$ and a velocity of $10 \mathrm{~km} \mathrm{~s}^{-1}$. Its abundances are solar.

We calculated the evolution of this system for two cases, one for a fast wind with normal, solar abundances, and one for a fast wind with [WC] abundances. These abundances are as listed in Table 1. The wind speed evolution is the same in both models, but the mass loss rate is taken to be 10 times higher in the [WC] case. The initial values (at a velocity of $25 \mathrm{~km} \mathrm{~s}^{-1}$ ) are $10^{-5} M_{\odot} \mathrm{yr}^{-1}$ for the [WC] star, and $10^{-6} M_{\odot} \mathrm{yr}^{-1}$ for the H-rich star. As pointed out above, the mass loss rate drops as the velocity increases. At the end of the calculation when the wind has a velocity of $2000 \mathrm{~km} \mathrm{~s}^{-1}$, the mass loss rates are $10^{-7}$ and $10^{-8} M_{\odot} \mathrm{yr}^{-1}$, respectively.

Figure 2 shows the evolution of the logarithm of the density as function of radius and time. Light shades correspond to higher densities. In this representation the evolution of the shell is easily traced. In both cases the bubble is initially momentumdriven and the inner shock and contact discontinuity lie close to each other, at the inner edge of the swept up shell. In the case of a fast wind with solar abundances, the inner shock detaches itself from the contact discontinuity at a time of 450 years, when the stellar wind has a velocity of $150 \mathrm{~km} \mathrm{~s}^{-1}$, and the shell has reached a radius of $7.5 \times 10^{-2} \mathrm{pc}$. This is in line with what was found by previous authors (Kahn \& Breitschwerdt 1990; Dwarkadas \& Balick 1998). In the case of the [WC] star, the transition happens at a much later time, 1100 years after the AGB, when the wind speed has reached $480 \mathrm{~km} \mathrm{~s}^{-1}$, at a radius of $2.2 \times 10^{-1} \mathrm{pc}$. Since the cooling time is proportional to the third power of the shock velocity, and inversely proportional to the density, this implies that the cooling is effectively 30 times higher (the effect of the density is largely cancelled due to the fact that the transition happens at a larger radius).

With the wind velocities mentioned above, we are reaching the regime where other elements such as Fe, can contribute substantially to the cooling. This could mean that the actual transition velocity for the solar abundances case is somewhat higher. However, our value is in line with what was found before, even with cooling curves including these heavier elements. Apparently, our omission is not so serious for this case. For the [WC] case, where the abundances of the heavier elements are mostly "normal", their contribution to the cooling is negligible compared to highly overabundant carbon.

The expansion velocity of the PN shell is found to be $50 \%$ higher in the case of the [WC] star, e.g. $21 \mathrm{~km} \mathrm{~s}^{-1}$ versus $17 \mathrm{~km} \mathrm{~s}^{-1}$ at $t=2000$ years. This is mostly due to the higher mass loss rate in the [WC] stellar wind.

We also ran simulations with a much steeper rise of the fast wind velocity, using $\alpha=2.5$ in Eq. (2). As was found in previous work (Kahn \& Breitschwerdt 1990; Dwarkadas \& Balick 1998), this does not affect the transition velocities. Obviously, the transition times do change. Since the behaviour of the postAGB wind is not well known, one cannot say much about the actual transition time, only about the transition velocity. 
Table 1. Abundances used in the various simulations.

\begin{tabular}{lllllll}
\hline \hline Element & Solar & {$[\mathrm{WC}]$} & WNl & WNe & WCl & WCe \\
\hline $\mathrm{H}$ & $9.21 \times 10^{-1}$ & $9.98 \times 10^{-3}$ & $1.51 \times 10^{-1}$ & $1.01 \times 10^{-6}$ & $9.89 \times 10^{-5}$ & $1.01 \times 10^{-4}$ \\
$\mathrm{He}$ & $7.83 \times 10^{-2}$ & $7.09 \times 10^{-1}$ & $8.38 \times 10^{-1}$ & $9.89 \times 10^{-1}$ & $6.43 \times 10^{-1}$ & $3.83 \times 10^{-1}$ \\
$\mathrm{C}$ & $3.05 \times 10^{-4}$ & $2.60 \times 10^{-1}$ & $1.01 \times 10^{-4}$ & $1.01 \times 10^{-4}$ & $2.77 \times 10^{-1}$ & $4.64 \times 10^{-1}$ \\
$\mathrm{~N}$ & $7.66 \times 10^{-5}$ & $9.98 \times 10^{-4}$ & $1.01 \times 10^{-2}$ & $1.01 \times 10^{-2}$ & $9.89 \times 10^{-5}$ & $1.01 \times 10^{-4}$ \\
$\mathrm{O}$ & $6.22 \times 10^{-4}$ & $2.00 \times 10^{-2}$ & $3.03 \times 10^{-4}$ & $3.03 \times 10^{-4}$ & $7.91 \times 10^{-2}$ & $1.51 \times 10^{-1}$ \\
$\mathrm{Ne}$ & $1.11 \times 10^{-4}$ & $9.98 \times 10^{-4}$ & $1.01 \times 10^{-4}$ & $1.01 \times 10^{-3}$ & $9.89 \times 10^{-4}$ & $1.01 \times 10^{-3}$ \\
\hline
\end{tabular}

Crowther, private communication.
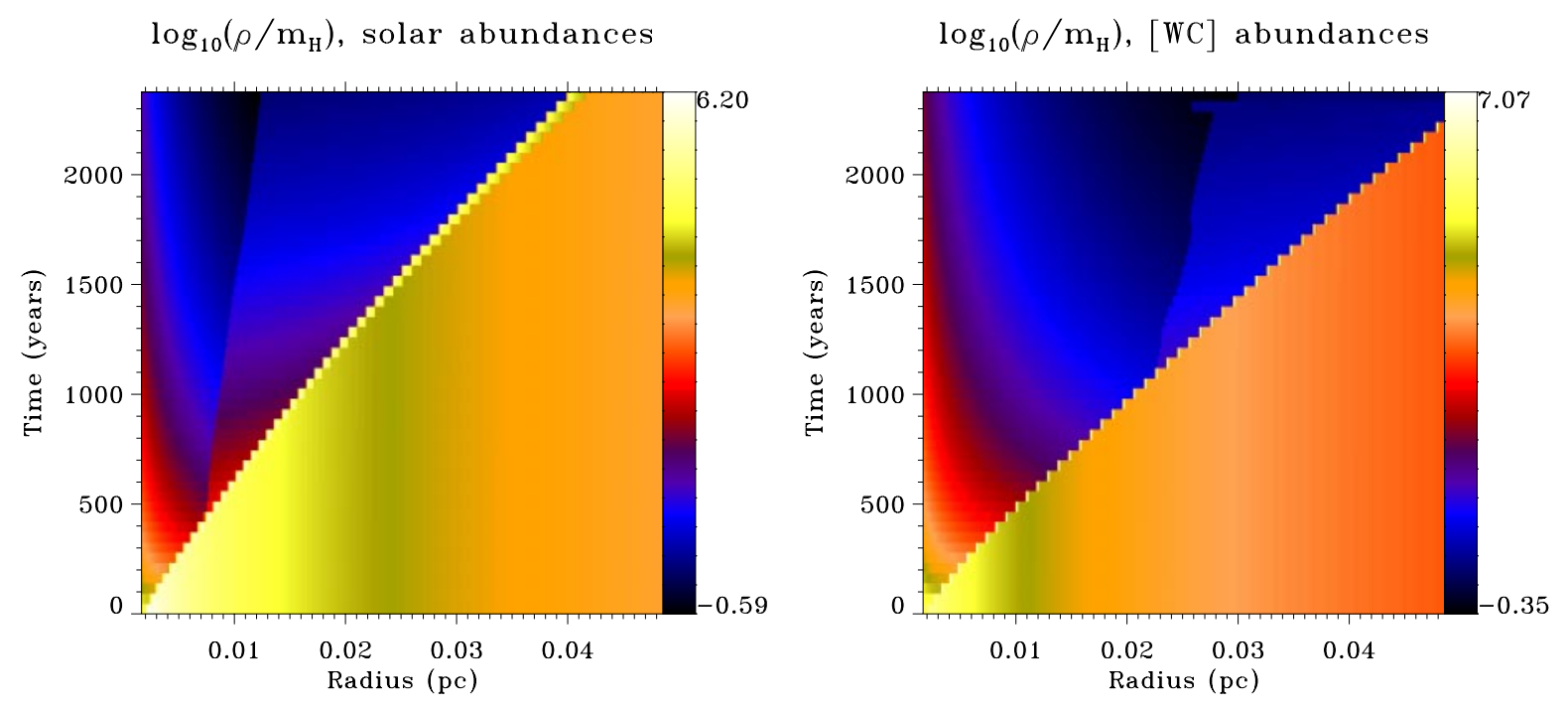

Fig. 2. Colour plot of $\log _{10}\left(\rho / m_{\mathrm{H}}\right)$ as function of radius and time for the two PN simulations.

This shows that the [WC] character does change the evolution of the SWB during the early phases of PN formation: higher mass loss rates will lead to higher expansion velocities and the high $\mathrm{C}$ abundance will increase the wind velocity at which the SWB changes from momentum- to energy-driven. The implications of this and the connection to observations are discussed in Sect. 6.

\section{Models for RNe}

For the RNe it is less easy to define one general model. First of all, the different types of WR stars have different abundances (see Table 1), where one distinguishes between nitrogen-rich WN and carbon-rich WC. Largely depending on the effective temperature, the subclass "l" for late, i.e. cooler, and "e" for early, i.e. hotter can be added.

The results from the previous section show that for WC abundances, a large effect can be expected. However, for the WN stars, the metals are an order of magnitude less abundant, helium being by far the most abundant element. Some stars are thought to evolve from WN into WC, so the abundances in those WR winds will change with time. The other complication is the mass loss history of these stars, as pointed out in the introduction.

In order to keep the numerical experiment simple, we run simulations of a WR wind running into a Red Super Giant
(RSG) wind, keeping the abundances in the WR wind constant. This problem then resembles that of the PNe, except that the mass loss rates are higher, and the wind speed is constant in time. This way we can study the effects of the H-deficient cooling on the bubble evolution.

We ran five simulations which only differed in the abundance of the stellar wind, one with solar abundances, and one for each type of WR star: WNl, WNe, WCl, WCe. All parameters are as listed in Table 2.

Figure 3 shows the evolution of the logarithm of the number density in the same way as in Fig. 2. One notices how the lowest velocity wind (WNl) model produces a momentumdriven RN until $t=550$ years, at a radius $7.2 \times 10^{-2} \mathrm{pc}$, after which it switches to energy-driven. The "solar", WNe, WCe, and $\mathrm{WCl}$ models are all energy-driven from the start, as is the WCe model (not shown).

Due to the low densities, collisional ionization at the inner shock takes some time, and there is transition zone where the ionic concentrations are out of equilibrium. This zone is quite narrow $\left(2 \times 10^{16} \mathrm{~cm}\right)$, and its existence does not have a major effect on the bubble dynamics or expected X-ray spectrum.

\subsection{Momentum and energy ratios}

Lamers \& Cassinelli (1999) briefly discuss the dynamics of SWBs in Chap. 12. There they point out that one can 
Table 2. Input parameters for the RNe models.

\begin{tabular}{llllll}
\hline \hline & Solar & WNl & WNe & WCe & WCl \\
\hline$\dot{M}_{\text {sw }}\left(\dot{M} \mathrm{yr}^{-1}\right)$ & $8 \times 10^{-5}$ & $8 \times 10^{-5}$ & $8 \times 10^{-5}$ & $8 \times 10^{-5}$ & $8 \times 10^{-5}$ \\
$v_{\text {sw }}\left(\mathrm{km} \mathrm{s}^{-1}\right)$ & 30 & 30 & 30 & 30 & 30 \\
$\mathcal{M}_{\mathrm{sw}}\left(\mathrm{mach}^{-1}\right)$ & 1.1 & 1.1 & 1.1 & 1.1 \\
$\dot{M}_{\mathrm{fw}}\left(\dot{M} \mathrm{yr}^{-1}\right)$ & $1.5 \times 10^{-5}$ & $3 \times 10^{-5}$ & $3 \times 10^{-5}$ & $1.5 \times 10^{-5}$ & $1 \times 10^{-5}$ \\
$v_{\mathrm{fw}}\left(\mathrm{km} \mathrm{s}^{-1}\right)$ & 2300 & 840 & 2060 & 2300 & 1400 \\
$\mathcal{M}_{\mathrm{fw}}(\mathrm{mach})$ & 20 & 20 & 20 & 20 \\
$r_{0}(\mathrm{~cm})$ & $5 \times 10^{15}$ & $5 \times 10^{15}$ & $5 \times 10^{15}$ & $5 \times 10^{15}$ & $5 \times 10^{15}$ \\
grid size & 3200 & 3200 & 3200 & 3200 & 3200 \\
\hline
\end{tabular}

$\log _{10}\left(\rho / \mathrm{m}_{\mathrm{H}}\right)$, Solar simulation

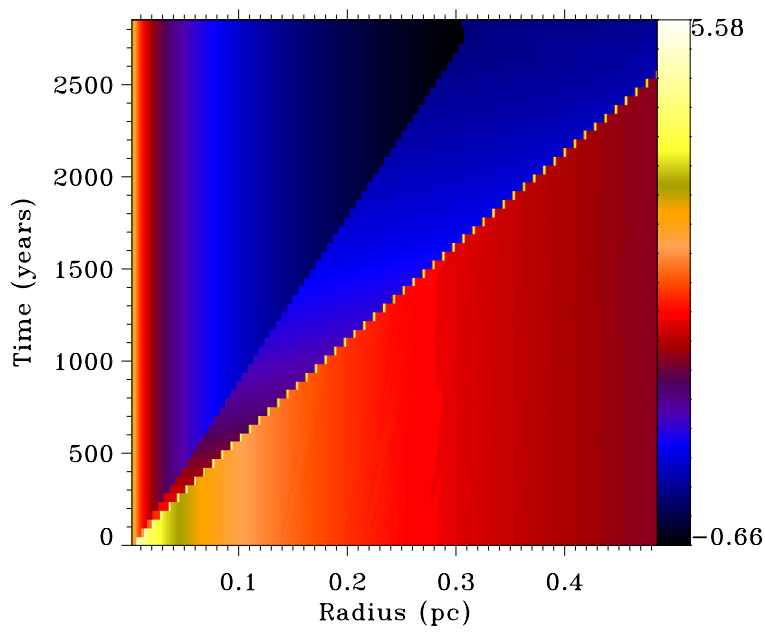

$\log _{10}\left(\rho / \mathrm{m}_{\mathrm{H}}\right)$, WNe simulation

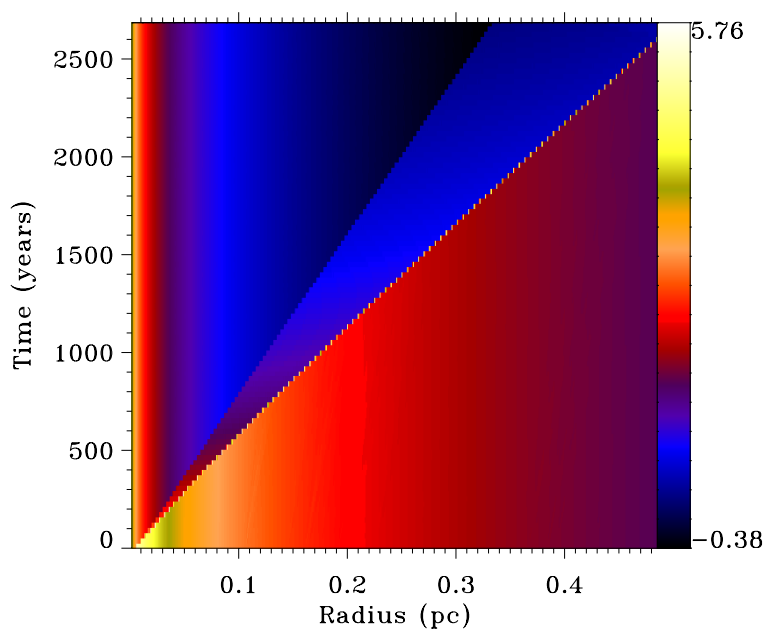

$\log _{10}\left(\rho / \mathrm{m}_{\mathrm{H}}\right)$, WCl simulation

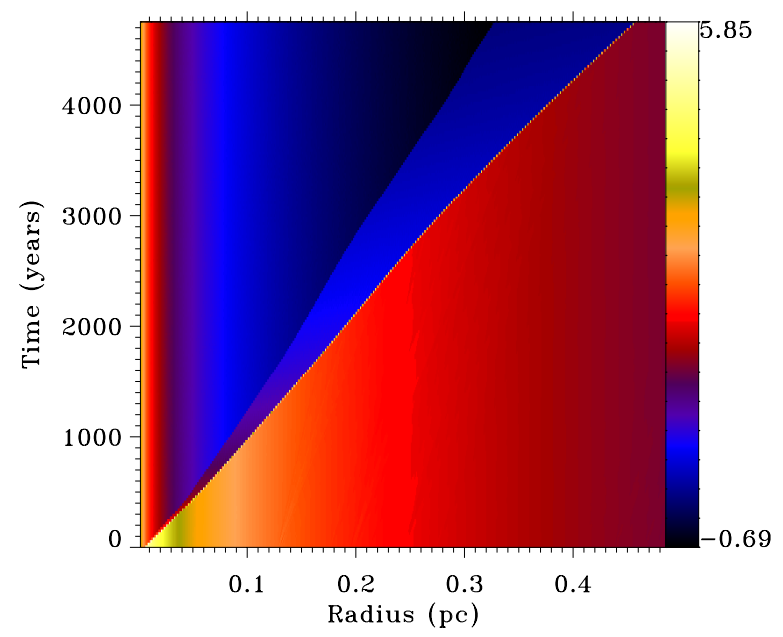

$\log _{10}\left(\rho / m_{H}\right)$, WNl simulation

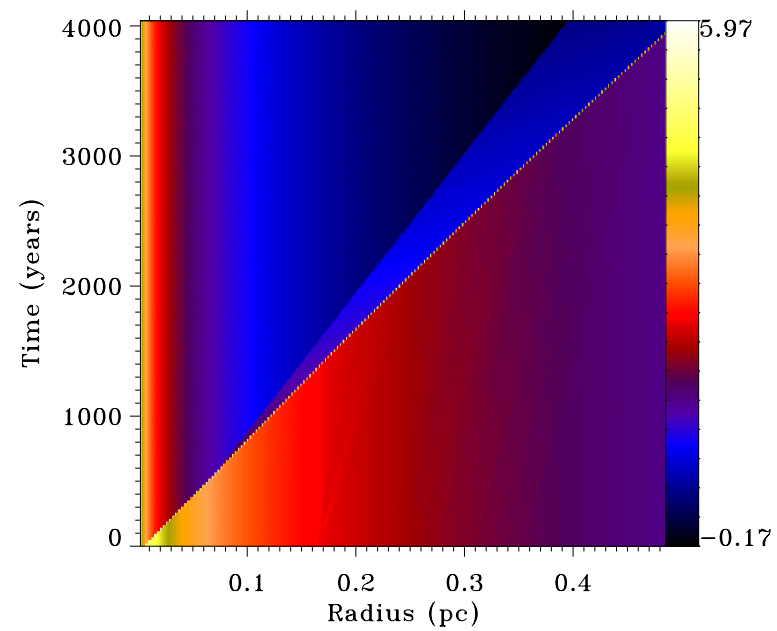

Fig. 3. Colour plot of $\log _{10}\left(\rho / m_{\mathrm{H}}\right)$ as function of radius and time for four of the five RN simulations.

determine whether a SWB is momentum-driven or energydriven by estimating the momentum and kinetic energy of the swept-up shell, and comparing this to the momentum and kinetic energy supplied by the stellar wind during the lifetime of the SWB. The ratio of shell momentum to wind momentum is called $\pi$, the ratio of the kinetic energies is $\epsilon$.

$$
\begin{aligned}
& \pi=\frac{M_{\text {shell }} v_{\text {shell }}}{\dot{M}_{\mathrm{fw}} v_{\mathrm{fw}} t} \\
& \epsilon=\frac{M_{\mathrm{shell}} v_{\mathrm{shell}}^{2}}{\dot{M}_{\mathrm{fw}} v_{\mathrm{fw}}^{2} t} .
\end{aligned}
$$


Garcia-Segura \& Mac Low (1995) derived an analytical description for SWBs around WR stars, including expressions for the ratios $\pi$ and $\epsilon$. However, they assumed that $v_{\mathrm{sw}}$ can be taken to be zero. While it is true that for WR stars $v_{\mathrm{sw}} \ll v_{\mathrm{fw}}$, often $\dot{M}_{\mathrm{fw}} v_{\mathrm{fw}} \sim \dot{M}_{\mathrm{sw}} v_{\mathrm{sw}}$, meaning that one should take the original momentum of the slow wind into account. Also, $v_{\mathrm{sw}}$ can be a sizeable fraction of $v_{\text {shell }}$, which means that the original velocity of the slow wind can be a non-negligible fraction of the shell velocity.

Using a thin-shell approximation, the expansion velocity of the shell can be found by considering momentum- and energy conservation. For an energy-driven bubble, momentum conservation means that

$\frac{\mathrm{d}}{\mathrm{d} t}\left[M_{\text {shell }}\left(\dot{r}-v_{\mathrm{sw}}\right)\right]=4 \pi r^{2} P$,

where $P$ is the pressure of the hot bubble. This pressure is related to input wind energy through energy conservation

$\frac{\mathrm{d}}{\mathrm{d} t}\left(\frac{4 \pi r^{3}}{3} \frac{P}{(\gamma-1)}\right)=\frac{1}{2} \dot{M}_{\mathrm{fw}} v_{\mathrm{fw}}^{2}-4 \pi r^{2} P \frac{\mathrm{d} r}{\mathrm{~d} t}$,

where the radius of the inner shock is assumed to be much smaller than the radius of the shell. The mass of the swept up shell $\left(M_{\text {shell }}\right)$ at a time $t$, when the shell has reached a radius $r=v_{\text {exp }} t$, is given by

$$
\begin{aligned}
M_{\text {shell }} & =\int_{0}^{r-v_{\mathrm{sw}} t} 4 \pi r^{2} \rho_{0} \frac{r_{0}^{2}}{r^{2}} \mathrm{~d} r \\
& =4 \pi r_{0}^{2} \rho_{0}\left(r-v_{\mathrm{sw}} t\right)=\frac{\dot{M}_{\mathrm{sw}}}{v_{\mathrm{sw}}}\left(r-v_{\mathrm{sw}} t\right),
\end{aligned}
$$

where $\dot{M}_{\mathrm{sw}}=4 \pi r_{0}^{2} \rho_{0} v_{\mathrm{sw}}$, with $r_{0}$ the radius at which the slow wind density is $\rho_{0}$.

Equations (5)-(7) can be combined into one equation of motion for the shell

$\frac{\dot{M}_{\mathrm{sw}}}{4 v_{\mathrm{sw}}} \frac{\mathrm{d}}{\mathrm{d} t}\left[r^{3} \frac{\mathrm{d}^{2}}{\mathrm{~d} t^{2}}\left(r-v_{\mathrm{sw}} t\right)^{2}\right]=\frac{1}{2} \dot{M}_{\mathrm{fw}} v_{\mathrm{fw}}^{2}$.

The solution of this equation is a shell expanding with constant velocity,

$$
\begin{aligned}
v_{\text {exp }, \mathrm{e}}= & v_{\mathrm{sw}}+v_{*, \mathrm{e}}\left[\left(\frac{1}{2}\left(1+\sqrt{1-4\left(\frac{v_{\mathrm{sw}}}{3 v_{*, \mathrm{e}}}\right)^{3}}\right)-\left(\frac{v_{\mathrm{sw}}}{3 v_{*, \mathrm{e}}}\right)^{3}\right)^{1 / 3}\right. \\
& \left.+\left(\frac{1}{2}\left(1-\sqrt{1-4\left(\frac{v_{\mathrm{sw}}}{3 v_{*, \mathrm{e}}}\right)^{3}}\right)-\left(\frac{v_{\mathrm{sw}}}{3 v_{*, \mathrm{e}}}\right)^{3}\right)^{1 / 3}\right]
\end{aligned}
$$

where

$$
v_{*, \mathrm{e}}=\left(\frac{1}{3} \frac{\dot{M}_{\mathrm{fw}}}{\dot{M}_{\mathrm{sw}}} v_{\mathrm{fw}}^{2} v_{\mathrm{sw}}\right)^{1 / 3}
$$

the solution for inifinitely small $v_{\mathrm{sw}}$, which can be found in for example Garcia-Segura \& Mac Low (1995) (Eq. (30)). Neglecting terms of order $\left(v_{\mathrm{sw}} / 3 v_{*, \mathrm{e}}\right)^{3}$, Eq. (9) can be approximated as

$v_{\mathrm{exp}, \mathrm{e}} \simeq v_{\mathrm{sw}}+v_{*, \mathrm{e}}$.
For the momentum conserving case it is easier to consider the balance between input momentum and shell momentum. The shell now consists of compressed material from both the slow and fast winds. The mass of swept up slow wind material which we will call $M_{\text {sh,sw }}$ is still given by Eq. (7), the mass of the fast wind material compressed into a shell at a position $r$ at a time $t$ is given by

$M_{\mathrm{sh}, \mathrm{fw}}=\frac{\dot{M}_{\mathrm{fw}}}{v_{\mathrm{fw}}}\left(v_{\mathrm{fw}} t-r\right)$,

i.e. the amount of mass supplied by the fast wind in a time $t$, minus the material which has not joined the shell yet.

Conservation of momentum says that the current momentum of the shell should equal the momentum added by the fast and slow winds:

$\left(M_{\mathrm{sh}, \mathrm{fw}}+M_{\mathrm{sh}, \mathrm{fw}}\right) v_{\mathrm{sh}}=M_{\mathrm{sh}, \mathrm{fw}} v_{\mathrm{fw}}+M_{\mathrm{sh}, \mathrm{fw}} v_{\mathrm{sw}}$.

Substituting Eqs. (7) and (12) into this expression and some algebra, shows that the solution is a shell with constant expansion velocity

$v_{\text {exp }, \mathrm{m}}=\frac{v_{\mathrm{sw}}+v_{*, \mathrm{~m}}}{1+v_{*, \mathrm{~m}} / v_{\mathrm{fw}}}$,

where

$v_{*, \mathrm{~m}} \equiv \sqrt{\frac{\dot{M}_{\mathrm{fw}}}{\dot{M}_{\mathrm{sw}}} v_{\mathrm{fw}} v_{\mathrm{sw}}}$,

the solution for $\dot{M}_{\mathrm{fw}} / \dot{M}_{\mathrm{sw}} \rightarrow 0$ (disregarding the mass contribution of the fast wind), and infinitely small $v_{\mathrm{sw}}$ (disregarding the slow wind's momentum input). This was the expression effectively used by Garcia-Segura \& Mac Low (1995).

Only dropping the terms linear or higher in $\dot{M}_{\mathrm{fw}} / \dot{M}_{\mathrm{sw}}$ and $v_{\mathrm{sw}} / v_{\mathrm{fw}}$, one obtains the approximate solution

$v_{\mathrm{exp}, \mathrm{m}} \simeq v_{\mathrm{sw}}+v_{*, \mathrm{~m}}$,

(see e.g. Kahn \& Breitschwerdt 1990, Eq. (16))

Using these new expressions for the expansion velocities, Eqs. (9) and (14), and assuming a radiative outer shock so that $v_{\text {shell }}=v_{\text {exp }}$, one can easily derive general expressions for the ratios $\pi$ and $\epsilon$. Since the expression for the expansion velocity in the energy-driven case is quite complicated, we will use the approximate expression Eq. (11) to derive a first order approximation for $\pi$ and $\epsilon$ in case of a non-zero slow wind velocity. This solution is valid as long as $\dot{M}_{\mathrm{fw}} v_{\mathrm{fw}}^{2} \ll \dot{M}_{\mathrm{sw}} v_{\mathrm{sw}}^{2}$. We thus obtain

$\pi_{\mathrm{e}} \simeq \frac{1}{3} \frac{v_{\mathrm{fw}}}{v_{*, \mathrm{e}}}+\frac{1}{3} \frac{v_{\mathrm{fw}} v_{\mathrm{sw}}}{\left(v_{*, \mathrm{e}}\right)^{2}}$

$\epsilon_{\mathrm{e}} \simeq \frac{1}{3}+\frac{1}{3} \frac{v_{\mathrm{sw}}}{v_{*, \mathrm{e}}}\left(2+\frac{v_{\mathrm{sw}}}{v_{*, \mathrm{e}}}\right)$.

For the momentum-driven case, the exact ratios are

$$
\begin{aligned}
\pi_{\mathrm{m}}= & \frac{v_{\mathrm{fw}}}{v_{\mathrm{fw}}+v_{*, \mathrm{~m}}}+v_{\mathrm{sw}} \frac{v_{\mathrm{fw}}-v_{*, \mathrm{~m}}-v_{\mathrm{sw}}}{v_{*, \mathrm{~m}} v_{\mathrm{fw}}+v_{*, \mathrm{~m}}^{2}} \\
\epsilon_{\mathrm{m}}= & \frac{v_{\mathrm{fw}} v_{*, \mathrm{~m}}}{\left(v_{\mathrm{fw}}+v_{*, \mathrm{~m}}\right)^{2}} \\
& +v_{\mathrm{sw}} \frac{v_{*, \mathrm{~m}}\left(2 v_{\mathrm{fw}}-v_{*, \mathrm{~m}}\right)+v_{\mathrm{sw}}\left(v_{\mathrm{fw}}-2 v_{*, \mathrm{~m}}-v_{\mathrm{sw}}\right)}{v_{*, \mathrm{~m}}\left(v_{\mathrm{fw}}+v_{*, \mathrm{~m}}\right)^{2}} .
\end{aligned}
$$


Table 3. Momentum and kinetic energy ratios for RNe models.

\begin{tabular}{llllll}
\hline \hline & Solar & WNl & WNe & WCe & WCl \\
\hline$\pi$ & 2.4 & 1.4 & 1.5 & 1.02 & 0.9 \\
$\pi_{\mathrm{e}}$ & 4.1 & 2.5 & 3.1 & 4.1 & 4.2 \\
$\pi_{\mathrm{m}}$ & 1.2 & 1.1 & 1.1 & 1.2 & 1.3 \\
$\epsilon$ & 0.21 & 0.20 & 0.16 & 0.056 & 0.06 \\
$\epsilon_{\mathrm{e}}$ & 0.43 & 0.49 & 0.42 & 0.43 & 0.50 \\
$\epsilon_{\mathrm{m}}$ & 0.07 & 0.15 & 0.09 & 0.07 & 0.09 \\
\hline
\end{tabular}

Taking $v_{\mathrm{sw}}=0$, and dropping terms of order $v_{*} / v_{\mathrm{fw}}$, one can recover the expressions from Garcia-Segura \& Mac Low (1995), their Eqs. (83) and (87).

According to Lamers \& Cassinelli (1999), RNe are mostly momentum-driven. This is mostly based on the work of Treffers \& Chu (1982) and Chu (1982), who derived momenta and kinetic energies for a set of $\mathrm{RNe}$, and found small values for $\pi$ and $\epsilon$, typically $\pi \sim 0.5$ and $\epsilon \sim 0.01$. More recent work, for example of Cappa et al. (1996), gives similar results for other nebulae.

Van Buren (1986) recalculated $\pi$ and $\epsilon$ for the five RNe from Chu (1982). He used the same data, but applied a correction for an unseen neutral component. He then finds larger values for both $\pi$ and $\epsilon$ : $\pi$ ranges from 1.2 to $6.8 ; \epsilon$ ranges from 0.013 to 0.15 . These values would make the SWBs energyconserving rather than momentum-conserving.

Observationally, one can think of a number of other complications when determining $\pi$ and $\epsilon$, such as determination of the mass and velocity of a clumpy flow, non-sphericity effects, unknown mass loss history, estimating the age of the RN, etc. As shown above, if the $\mathrm{RN}$ is expanding into material from a previous slower wind, one would also need an estimate of its velocity $v_{\mathrm{sw}}$.

This raises the question whether observationally derived values for $\pi$ and $\epsilon$ can be used to derive the character of the RNe.

In order to better evaluate the observationally derived values, we extract values of $\pi$ and $\epsilon$ from our simulations. In order to check that our procedure works, we determined $\pi$ and $\epsilon$ for two simulations in which the radiative source terms were switched off, but which used adiabatic indices $\gamma=5 / 3$ (energy-conserving) and $\gamma=1.001$ (momentum-conserving), respectively. The results from these simulations reproduce the analytical values from Eqs. (17)-(20) within 5\%.

In Table 3 we list the values found from the RN simulations, and the one expected from Eqs. (17)-(20). What one sees is that even though the SWBs are all energy-driven, in the sense that they contain hot shocked fast wind material, the values are lower than expected. This means that the partially radiative character of the SWB reduces the values for $\pi$ and $\epsilon$, and approach the values for momentum-driven bubbles. For the two WC simulations, the value actually drops below the ones expected for the momentum-driven case. This is because the swept-up material cools below its pre-shock temperature.
The cooling is more efficient here, due to mixing in of wind material into the shell.

These results shows that the ratios $\pi$ and $\epsilon$ are of very limited use when establishing the character of SWBs: an energydriven bubble may disguise itself as a momentum-driven bubble.

\section{Discussion}

\section{1. $\mathrm{PNe}$}

The effects of the [WC] wind in PNe may seem limited, only postponing the transition from momentum- to energy-driven bubbles to a later time. However, this later transition may have some serious consequences. First of all, during the momentumdriven phase the (proto-)PN suffers from the non-linear thin shell instability, (see e.g. Dwarkadas \& Balick 1998). A later transition gives this instability a longer time to work on the structure of the nebula. The result could then be a more disturbed or fragmented nebula. There are some observational indications for more substructure in [WR]-PNe (Górny 2001). More quantitatively, analysis of the emission lines from a number of [WR]-PNe shows that these lines are quite wide, sometimes wide enough to hide any splitting of the lines (Acker et al. 2002). To reproduce these line shapes, the authors invoke a turbulent velocity component, something which is not needed for normal PNe. This could be an effect of the later transition.

Because of the width of the lines, finding the expansion velocity of the nebulae becomes somewhat difficult. Earlier results indicated higher than average expansion velocity for [WR]-PNe (Górny 2001), but Acker et al. (2002) dispute this. Our models do predict a higher average expansion velocity, mostly due to the higher mass loss rates. Of course there will be large variations, depending on the precise mass loss history of the star, the age of the nebula, and the lines used to measure the expansion velocity.

If the transition from momentum- to energy-driven takes longer for [WR]-PNe, there is another interesting effect. During the momentum-driven phase, asphericities in the fast wind are easily imprinted on the swept-up shell. As the hot bubble develops, it forms more and more of a buffer between angular variations in the fast wind and the swept-up shell, and the aspherical fast wind has less and less of an effect on the shape of the nebula. If PNe acquire most of their asphericity due to an aspherical fast wind, one would therefore expect [WR]-PNe, which develop a hot bubble later, to be more aspherical. Since there seems to be no systematic morphological differences between [WR]-PNe and normal PNe (Górny 2001), the conclusion would be that aspherical post-AGB winds do not play a major role in determining the asphericity of PNe. This rather qualitative statement will be studied in some more detail in a subsequent paper.

The fact that the [WR]-PNe form a subgroup with clearly different mass loss properties may allow further tests of models for the formation of $\mathrm{PNe}$ in general. For instance, the efficiency 
of the magnetic shaping mechanism of Chevalier \& Luo (1994) is given by

$\sigma=\frac{B_{\mathrm{star}}^{2} R_{\mathrm{star}}^{2}}{\dot{M} v_{\mathrm{fw}}}\left(\frac{v_{\mathrm{rot}}}{v_{\mathrm{fw}}}\right)^{2}$,

where $B_{\text {star }}$ is the magnetic field strength at the surface of the star, $R_{\text {star }}$ is the stellar radius, $v_{\text {rot }}$ is the stellar rotation velocity. If typical field strengths and rotation speeds for [WR] central stars are the same as for normal central stars, the higher mass loss rates for [WR] stars implies lower values of $\sigma$, i.e. lesser efficiency of the magnetic shaping. This would then give on average rounder [WR]-PNe. The magnitude of the effect is about a factor 10. The uncertainties in the other key parameters $\left(B_{\text {star }}\right.$, $\left.v_{\text {rot }}\right)$, may make it hard to find this effect in the observations. However, in all models the formation of a PN is basically driven by the post-AGB wind, and that there are clear differences between the winds of [WC] and normal central stars, a closer comparison of the shapes of [WC]-PNe to normal PNe is warranted.

\section{2. $\mathrm{RNe}$}

The numerical models presented in the previous section show that it is unlikely that RNe around WR stars are momentumdriven. The amount of energy produced in shocking the fast wind cannot efficiently be radiated away, not even by the extremely metal-rich gas of the WR wind. Unless there are other physical mechanisms at work, the RNe cannot be momentumdriven.

A number of physical mechanisms may alter the results of our simplified models. Photo-ionization will alter the ionization state of parts of the RNe. However, the spectrum of WR stars is not hard enough to produce the ions which are collisionally generated in the hot bubble, and hence it will not change our conclusion about the energy-driven character of RNe. Clumpiness and time variability of the stellar wind will produce areas of different temperatures when shocked. However, the lower density portions of the wind will cool slowly, and are likely to take up the largest volume, so the bubble will still be filled with a substantial amount of hot gas, making it energy-driven. Clumpiness in the environment is likely to be more effective in modifying the character of the RNe. If these clumps are not effectively accelerated by the outer shock, they may end up inside the SWB, and start to evaporate, raising the density, and lowering the temperature of the hot shocked wind material, a process referred to as "mass loading". This could conceivably lead to more efficient cooling and a collapse of the hot bubble against the inner edge of the shell. Thermal conduction, if not inhibited by the presence of a magnetic field, can produce a similar result. However, studies of mass loading (Pittard et al. 2001) show no indication for such a collapse.

The observed X-ray spectra for RNe show the presence of hot gas (Wrigge et al. 1998; Wrigge 1999), although not as hot as predicted by the models. This is a strong observational indication that RNe are filled with hot gas, and are therefore energy-driven.

The remaining question is how to interpret the observationally derived values for $\pi$ and $\epsilon$. Our simulations show that these are of limited value. Even in the idealized case studied here, $\pi$ and $\epsilon$ do not follow the simple division between momentumand energy-driven. The observational problems are also many, as was already outlined by Van Buren (1986). In addition the complex and fast evolution of these stars and their winds, invalidates the assumption that one can simply integrate over time. The momentum and kinetic energy of the shells are an integration over the momenta and kinetic energy of the swept-up material, which could come from the interstellar medium and various previous mass loss phases. Dividing this by a value derived from assuming the current wind to have been constant in time, is unlikely to give sensible values.

\section{Conclusions}

We simulated the effects $\mathrm{H}$-deficient winds have on their SWBs, studying simplified cases for PNe and RNe. We find that the extreme abundances in the winds of [WC] stars can keep their PNe momentum-driven for a longer time. We speculate that this leads to more turbulent nebulae and would also produce more aspherical $\mathrm{PNe}$ if their shape was mostly due to an aspherical post-AGB wind.

For the RNe around massive WR stars, we showed that they cannot be momentum-driven, despite earlier claims to the opposite. We pointed out some of the difficulties related to the deriving the momentum- versus energy-driven character of the $\mathrm{RNe}$ using the comparisons of RN momentum and kinetic energy to the assumed total input of momentum and kinetic energy by the stellar wind.

The models in this article illustrate the physical effects of abundances on the structure of SWBs. To be realistic models for PNe and RNe, they should be improved in several ways. As shown for instance in Mellema (1994), ionization fronts can play an important role in the early evolution of PNe. For the $\mathrm{RNe}$ adding photo-ionization to the models could help in estimating the amount of neutral material, which is one of the unknowns in the $\pi-\epsilon$ method. Letting their winds evolve according to a more realistic recipe such as in Mellema (1994) for PNe, and in Garcia-Segura et al. (1996a) for RNe, would be another step in the direction of more realistic models. However, given that the results in this paper show that for RNe the wind abundances play only a minor role, the models of Garcia-Segura et al. (1996b) and Garcia-Segura et al. (1996a) remain largely valid, and only for the case of the [WR]-PNe it makes sense to pursue more realistic models.

Acknowledgements. This work was sponsored by the Stichting Nationale Computerfaciliteiten (National Computing Foundation, $\mathrm{NCF}$ ) for the use of supercomputer facilities, with financial support from the Nederlandse Organisatie voor Wetenschappelijk Onderzoek (Netherlands Organization for Scientific Research, NWO). The research of GM has been made possible by a fellowship of the Royal Netherlands Academy of Arts and Sciences. PL is a Reserach Fellow at the Royal Swedish Academy supported by a grant from the Wallenberg Foundation. GM and PL acknowledge support from the Swedish Research Council for funding working visits between Stockholm and Leiden. We thank the referee (R. Chevalier) for useful comments and pointing out an error in the original version of this paper. 


\section{References}

Abbott, D. C., \& Conti, P. S. 1987, ARA\&A, 25, 113

Acker, A., Gesicki, K., Grosdidier, Y., \& Durand, S. 2002, A\&A, 384, 620

Balick, B., Mellema, G., \& Frank, A. 1993, A\&A, 275, 588

Cappa, C. E., Niemela, V. S., Herbstmeier, U., \& Koribalski, B. 1996, A\&A, 312, 283

Chevalier, R. A., \& Luo, D. 1994, ApJ, 421, 225

Chu, Y.-H. 1982, ApJ, 254, 578

Cox, D. P., \& Daltabuit, E. 1971, ApJ, 167, 113

Cox, D. P., \& Tucker, W. H. 1969, ApJ, 157, 1157

Dalgarno, A., \& McCray, R. A. 1972, ARA\&A, 10, 375

Dwarkadas, V. V., \& Balick, B. 1998, ApJ, 497, 267

Dyson, J. E., \& Williams, D. A. 1997, The physics of the interstellar medium, 2nd ed. (Institute of Physics Publishing, ISBN 0750303069)

Frank, A., \& Mellema, G. 1994, A\&A, 289, 937

Górny, S. K. 2001, Ap\&SS, 275, 67

Gaetz, T. J., \& Salpeter, E. E. 1983, ApJS, 52, 155

Garcia-Segura, G., Langer, N., \& Mac Low, M.-M. 1996a, A\&A, 316, 133

Garcia-Segura, G., \& Mac Low, M. 1995, ApJ, 455, 145

Garcia-Segura, G., Mac Low, M.-M., \& Langer, N. 1996b, A\&A, 305, 229

Gould, R. J. 1980, ApJ, 238, 1026
Gronenschild, E. H. B. M., \& Mewe, R. 1978, A\&AS, 32, 283

Hamann, W.-R. 2002, in IAU Symp. 209, Planetary Nebulae, in press Herwig, F. 2001, Ap\&SS, 275, 15

Kahn, F. D., \& Breitschwerdt, D. 1990, MNRAS, 242, 505

Karzas, W. J., \& Latter, R. 1961, ApJS, 6, 167

Koesterke, L. 2001, Ap\&SS, 275, 41

Koo, B., \& McKee, C. F. 1992a, ApJ, 388, 93

Koo, B., \& McKee, C. F. 1992b, ApJ, 388, 103

Lamers, H. J. G. L. M., \& Cassinelli, J. P. 1999, Introduction to stellar winds (Cambridge University Press, ISBN 0521593980)

LeVeque, R. J. 2002, Finite Volume Methods for Hyperbolic Problems (Cambridge University Press, ISBN 0521009243)

Mellema, G. 1994, A\&A, 290, 915

Mellema, G., Eulderink, F., \& Icke, V. 1991, A\&A, 252, 718

Mellema, G., Raga, A. C., Canto, J., et al. 1998, A\&A, 331, 335

Pittard, J. M., Hartquist, T. W., \& Dyson, J. E. 2001, A\&A, 373, 1043

Plewa, T., \& Müller, E. 1999, A\&A, 342, 179

Raga, A. C., Mellema, G., \& Lundqvist, P. 1997, ApJS, 109, 517

Raymond, J. C., Cox, D. P., \& Smith, B. W. 1976, ApJ, 204, 290

Roe, P. 1981, J. Comp. Phys., 43, 357

Sutherland, R. S., \& Dopita, M. A. 1993, ApJS, 88, 253

Treffers, R. R., \& Chu, Y.-H. 1982, ApJ, 254, 569

Van Buren, D. 1986, ApJ, 306, 538

Wrigge, M. 1999, A\&A, 343, 599

Wrigge, M., Chu, Y.-H., Magnier, E. A., \& Kamata, Y. 1998, in Physics (Berlin Springer Verlag Lecture Notes), 506, 425 